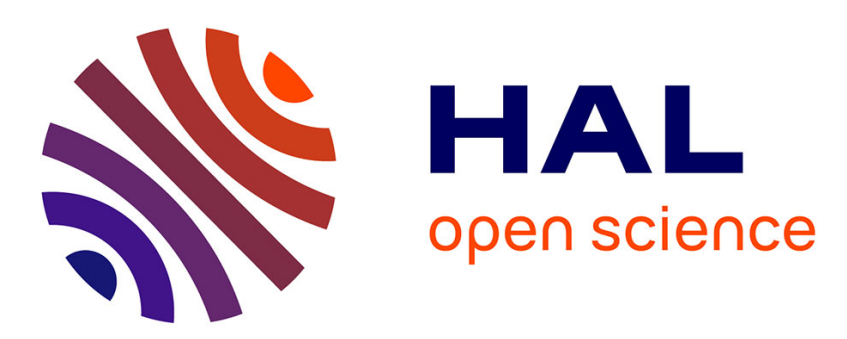

\title{
Integrated automated vehicle string longitudinal control
}

Saïd Mammar, Naima Ait Oufroukh, Lydie Nouveliere, Dominique Gruyer

\section{To cite this version:}

Saïd Mammar, Naima Ait Oufroukh, Lydie Nouveliere, Dominique Gruyer. Integrated automated vehicle string longitudinal control. IEEE Intelligent Vehicles Symposium (IEEE IV 2013), Jun 2013, Gold Coast, Australia. pp.803-808, 10.1109/IVS.2013.6629565 . hal-00941317

\section{HAL Id: hal-00941317 https://hal.science/hal-00941317}

Submitted on 12 Jul 2021

HAL is a multi-disciplinary open access archive for the deposit and dissemination of scientific research documents, whether they are published or not. The documents may come from teaching and research institutions in France or abroad, or from public or private research centers.
L'archive ouverte pluridisciplinaire HAL, est destinée au dépôt et à la diffusion de documents scientifiques de niveau recherche, publiés ou non, émanant des établissements d'enseignement et de recherche français ou étrangers, des laboratoires publics ou privés. 


\title{
Integrated Automated Vehicle String Longitudinal Control
}

\author{
Saïd Mammar, Naïma Ait Oufroukh, Lydie Nouvelière, Dominique Gruyer
}

\begin{abstract}
This paper presents the design and simulation test of an automated vehicle string longitudinal control. The vehicle string is constituted of three highly cooperative vehicles following a leader. The string follows a leading vehicle which is supposed manually driven or at least not cooperative. The first vehicle of the string uses only its on-board sensor to obtain relative speed and inter-distance to the leader. Based on Lyapunov theory and invariant sets, the control law of the string is able to guarantee that the state trajectories remain bounded under bounded disturbance inputs, such as leading vehicle acceleration. The control law is designed in such a way that variable time headway strategy is naturally handled. The control law is computed using Linear and Bilinear Matrix Inequalities (LMI-BMI) methods. Some design parameters can be adjusted to handle the trade-off between safety constraints and comfort specifications.
\end{abstract}

\section{INTRODUCTION}

Vehicle longitudinal control has been the object of intensive research over the last decades [1]. After cruise control, which is now widely implemented on vehicles, research has shifted to the adaptive cruise control (ACC) which is an extension of the cruise control that manages both relative speed and distance to a preceding vehicle [2]. Starting just before the new millennium, ACC is already available not only in luxury passenger cars and trucks but also recently in some compact cars. In addition, vehicles will be shortly equipped with communication means.

Nowadays, urban highways experience recurrent congestion. One could then gain road capacity from smoothing traffic behavior while reducing driver workload and fuel consumption if vehicles are organized in strings or platoons. These facts have been shown in previous work on automated platoons [3], [4], [5]. European Framework 7 programme SARTRE has also addressed the development of strategies and technologies to allow vehicle platoons to operate on normal public highways with significant environmental, safety and comfort benefits [6]. In [1], different spacing policies have been cited in order to enhance both safety and capacity. In [7], vehicle longitudinal control has been considered using a reference model inspired from a non-linear damper/spring model associated with safety zones. Time headway based spacing appears as the most promising strategy. It is currently implemented on commercial vehicles equipped with ACC,

S. Mammar, N. Ait Oufroukh and L. Nouvelière are with Université d'Évry Val d'Essonne, France. IBISC: Informatique, Biologie Intégrative et Systèmes Complexes - EA 4526, 40 rue du Pelvoux CE1455, 91020, Evry, Cedex, France, (e-mail: (said.mammar, naima.aitoufroukh, lydie.nouveliere)@ibisc.univ-evry.fr). D. Gruyer is with LIVICIFSTTAR, e-mail: dominique.gruyer@ifsttar.fr.

This work has been conducted as a part of the French national research project ANR-ABV. and it has been proved that it is natural for the driver. However, in steady state, time headway is directly related to lane capacity. Thus, from the road manager point of view, it would be appreciable that time headway could be tuned in order to achieve macroscopic traffic characteristics requirements. On the other hand vehicle communication and delay has been addressed in several works [8], [9], [10]. It has been shown that full communication mode allows satisfactory performances with controller synthesized with simplified models. In fact, as mentioned in [11], Longitudinal control systems typically consist of two parts: a vehicle-independent part and a vehicle-dependent part [12],[13]. The vehicle dependent part in the inner loops directly controls the actuator, in order to achieve the desired acceleration/deceleration generated by the vehicle-independent part.

This paper aims at developing an integrated controller for a string of three vehicles following a manually driven leader. In extend, this approach allows one vehicle to follow autonomously a leader using on-board sensors. If two or three vehicles equipped with communication means are in following mode, they could enter in a platoon mode where each of the vehicles uses the full state of the string for control. In order to make the system flexible, the control law achieves requirements of comfort and safety for a given range of headway time. When writing down the model, the leading vehicle acceleration enters as an unknown bounded disturbance input. From available car industry sensors, it is hard to estimate this variable. The aim of the proposed control is to ensure both vehicle following and collision avoidance. For this purpose, invariant sets framework, which has significantly developed in control engineering over the last decades, is used [14]. The existence of an invariant set (ellipsoidal or polyhedral set), under bounded disturbance, is equivalent to the "input-to state stability" (ISS) notion [15]. This framework ensures that every trajectory starting inside the invariant set will not exceed it [16].

The remainder of this paper is organized as follows. The assistance specifications are given in Section II including the description of the safety driving zones and a switching strategy between the driver and the assistance. Section III contains the description of the vehicle inter-distance model. The design of the longitudinal control assistance is further conducted in Section IV. Section V contains simulation results for several maneuvers including ACC and stop-andgo traffic. Conclusions are presented in Section VI. 


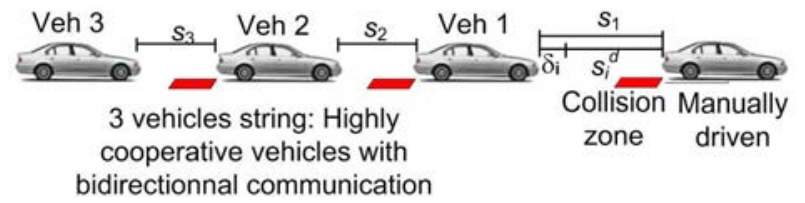

Fig. 1. Vehicle string setup.

\section{VEHICLE STRING INTEGRATED LONGITUdINAL CONTROL CONCEPT}

The main goal of the proposed control law is to achieve the longitudinal control of a string of vehicles under automated longitudinal control mode. The proposed concept is applicable for sub-urban longitudinal control particularly in dense traffic where vehicle following includes ACC mode and stop-and-go. The control scheme for a string of vehicles is plotted in Fig. 1. The lead vehicle is hand driven or at least in automatic mode but is not cooperative. The first follower vehicle (veh 1) uses only on-board sensing to acquire inter-distance and relative speed. He has no access to front vehicle acceleration. Follower vehicles (veh 1 to veh 3) are cooperative. For these vehicles, acceleration and braking tasks are carried out by on-board controllers that use information coming both from on-board sensors and communication systems. More specifically, the assistance should ensure vehicle following according to a chosen interdistance policy and it should avoid rear-end collisions and large overlap from the reference distance. The concept follows these main characteristics:

- The vehicle string is formed by at most three vehicles following a manually driven leader.

- No information is available from the leading vehicle unless those obtained from on-board sensors (video, lidar,...). This assumption ensures a more flexible implementation of the concept since even at few penetration rate, vehicles equipped with such a system can form a string and follow in ACC mode a conventional vehicle.

- Communication network within the string, unless the leader allows each vehicle to access to all vehicles acceleration, deviation from headway distances and relative speeds between all vehicles of the string.

- The reference distance could be based on variable time headway in order to allow lower or greater lane capacity when required by the infrastructure manager.

- The vehicles state variables deviation should be limited in order to avoid entering the collision zone.

- The state variables and the vehicle acceleration/brake have to be bounded to guarantee safety and comfort.This will be defined in more details in the next section.

\section{VEHICLE AND STRING MODELS}

\section{A. Vehicle model}

The acceleration of vehicle can be described by the following equation

$$
a=\frac{1}{m}\left[F_{e x}+\frac{1}{r_{e}}\left(T_{r r}+\tau_{e}+\tau_{b}\right)\right]
$$

where $m$ denotes vehicle mass, $F_{e x}$ stands for the external force which embeds the air drag and the gravitational force due to road slope, $r_{e}$ is the wheel effective radius, $T_{r r}$ is the rolling resistance torque, while $\tau_{e}$ and $\tau_{b}$ are the engine torque and, respectively, braking torque. Vehicle longitudinal dynamics are known to be highly nonlinear, particularly when including the engine dynamics. However, in all longitudinal control architectures, the control is performed in two loops: an inner loop which compensates the nonlinear vehicle dynamics and achieves the desired algebraic acceleration by acting on the throttle or on the brakes, and an outer loop which is responsible for guaranteeing good tracking of the desired vehicle inter-distance. This decomposition is also called vehicle dependent and vehicle independent control parts. The vehicle-dependent loop is beyond the scope of this paper. It is assumed that it has already been designed and we are only interested here in the vehicle-independent part. Further details could be found in [17]. In the reminder, the $i$-th vehicle of a string longitudinal dynamics, including actuators, is approximated by the following model

$$
\dot{a}_{i}(t)=-\frac{1}{\tau_{i}} a_{i}(t)+\frac{g_{i}}{\tau_{i}} u_{i}^{a}(t)
$$

where $a_{i}$ denotes the acceleration of vehicle $i, u_{i}^{a}$ its acceleration demand, $\tau_{i}$ and $g_{i}$ denote the actuators time constant and gain respectively. The two parameters vary from one vehicle to another. In addition, parameter $g_{i}$ depends on the current mode: braking or driving. In this paper, these parameters are considered as uncertain, their nominal values are $\tau_{i}=0.1 \mathrm{sec}$ and $g_{i}=1$. When the assistance is active, it is assumed that an x-by-wire system allows to cancel the driver demand inputs (acceleration and braking) and as a consequence the acceleration/braking demand is that delivered by the controller.

\section{B. Vehicle string model}

In the proposed concept, a string of vehicles is started as soon as a preceding vehicle appears in the range of the host vehicle sensor with a slower speed, forming a couple of two vehicles or a string of three vehicles (four with the leader). For leading vehicle with index $i=0$, the acceleration demand is assumed to be an external input.

Given the positions $x_{i-1}(t)$ and $x_{i}(t)$ of vehicles of index $i$ and $(i-1)$ respectively, the headway distance is $s_{i}=$ $x_{i-1}(t)-x_{i}(t)$ and the relative speed is $v_{i}^{r}=\dot{x}_{i-1}(t)-\dot{x}_{i}(t)$. The headway distance dynamics can be represented as a double integrator driven by the difference between the leader vehicle acceleration and the follower vehicle acceleration, i.e.,

$$
\dot{v}_{i}^{r}=\ddot{s}_{i}=a_{i-1}(t)-a_{i}(t)
$$

Several spacing strategies have been tested both for ACC systems and in the scope of the fully automated longitudinal control for automated highways. They include constant spacing, constant time headway and nonlinear time or space dependent headway. In this study, the control law aims at tracking a constant time headway distance. This interdistance policy is proportional to the host vehicle speed, $v_{i}(t)$, 
which leads to more spacing at higher speed. Desired spacing is thus given by $s_{i}^{d}(t)=s_{0}+h v_{i}(t)$, where $s_{0}$ is the minimum spacing at stopping and $h$ is the time headway.

The spacing error of the ith follower vehicle if defined by $\delta_{i}(t)=s_{i}(t)-s_{i}^{d}(t)$. It represents the difference between the actual spacing and the desired one. According to the previous equations, the final state-space model is given by:

$$
\dot{\delta}_{i}(t)=v_{i}^{r}(t)-h a_{i}(t)
$$

Combining equations (2)-(4), one can achieve for each vehicle the following spacing error dynamics

$$
\left[\begin{array}{c}
\dot{\delta}_{i} \\
\dot{v}_{i}^{r} \\
\dot{a}_{i}
\end{array}\right]=\bar{A}_{i}\left[\begin{array}{c}
\delta_{i} \\
v_{i}^{r} \\
a_{i}
\end{array}\right]+\bar{B}_{i} u_{i}^{a}+\bar{E}_{i} a_{i-1}
$$

with

$$
\bar{A}_{i}=\left[\begin{array}{ccc}
0 & 1 & -h \\
0 & 0 & -1 \\
0 & 0 & -\frac{1}{\tau_{i}}
\end{array}\right], \bar{B}_{i}=\left[\begin{array}{c}
0 \\
0 \\
\frac{g_{i}}{\tau_{i}}
\end{array}\right], \bar{E}_{i}=\left[\begin{array}{l}
0 \\
1 \\
0
\end{array}\right]
$$

In order to obtain a controller that is directly implementable on an Electronic Control Unit (ECU), the continuous-time state-space equation (6) is converted into a discrete-time model using a zero-order hold assumption on the inputs and an Euler discretization method with sample time $T_{s}=$ $0.01 s$. The sampling times $t=k T_{s}$, where $k$ represents the successive time steps belonging to $\mathscr{I}$, the set of integers. Vehicle longitudinal control needs to incorporate constraints on the absolute value of the jerk $j_{i}(t)$ of the host vehicle. In addition, an integral action in the open-loop ensures zero steady state error. Consequently, the model is converted into an incremental input-output model (IIO). The new control input is in this case $u_{i}(k)=\delta u_{i}^{a}(k)=u_{i}^{a}(k)-u_{i}^{a}(k-1)$ and the IIO model is obtained by extending the state to $x_{i}^{e}(k)=\left[\begin{array}{cccc}\delta_{i} & v_{i}^{r} & a_{i} & u_{i}^{a}(k-1)\end{array}\right]^{T}$

$$
x_{i}^{e}(k+1)=\overline{\bar{A}}_{i} x_{i}^{e}(k)+\overline{\bar{B}}_{i} u_{i}(k)+\overline{\bar{E}}_{i} a_{i-1}(k)
$$

where

$\overline{\bar{A}}_{i}=\left[\begin{array}{cccc}1 & T_{S} & -h T_{S} & 0 \\ 0 & 1 & -T_{S} & 0 \\ 0 & 0 & 1-\frac{1}{\tau_{i}} T_{S} & \frac{g_{i}}{\tau_{i}} T_{S} \\ 0 & 0 & 0 & 1\end{array}\right], \bar{B}_{i}=\left[\begin{array}{c}0 \\ 0 \\ \frac{g_{i}}{\tau_{i}} T_{S} \\ 1\end{array}\right], \overline{\bar{E}}_{i}=\left[\begin{array}{c}0 \\ T_{S} \\ 0 \\ 0\end{array}\right]$

The open loop model of a string is constructed by introducing the state vector $x^{T}=\left[\begin{array}{ccc}x_{1}^{e} & \cdots & x_{n}^{e}\end{array}\right] \in \mathscr{R}^{4 n}$, where $n=3$ is the number of vehicles in the string. The control input vector is $u^{T}=\left[\begin{array}{lll}u_{1} & \cdots & u_{n}\end{array}\right] \in \mathscr{R}^{n}$ and the input $a_{0}$ represents the leading vehicle acceleration which acts in this case as an unknown disturbance input. In addition, it is assumed that the leading vehicle acceleration is bounded, $\left|a_{0}\right| \leq a_{0}^{\max }$, one defines a normalized disturbance input $|w| \leq$ 1 such that $a_{0}=a_{0}^{\max } w$ and then the IIO model.

$$
x(k+1)=A x(k)+B u(k)+E w(k)
$$

The whole state vector is used for control synthesis. The variables are measured using on-board sensors or obtained through a communication system, according to the considered scenario.

In order to tackle with various traffic conditions, the headway time is assumed to be variable in the range $\left[h_{\min }, h_{\max }\right]$. Thus, matrix $A$ in system (9) is a function of $h$, namely $A(h)$. Therefore, the system could be converted into an affine LPV system with two vertices system obtained for the extremal values of $h$. One can write:

$$
x_{e}(k+1)=\sum_{i=1}^{2} \mu_{i} A_{i} x(k)+B u(k)+E w(k)
$$

where $A_{1}=A\left(h_{\min }\right), A_{2}=A\left(h_{\max }\right)$. The coefficients $\mu_{1}$ and $\mu_{2}$ are positive and verify equations $\left(\mu_{1}+\mu_{2}=1\right)$ and $\left(\mu_{1} h_{\min }+\mu_{2} h_{\max }=h\right)$, which determine the parameters. Note that the matrix $B$ is parameter independent. This simplifies the controller synthesis as single summation index is only required.

\section{CONTROL SYNTHESIS}

Our aim is now to synthesize an LPV state feedback of the form

$$
u(k)=\sum_{i=1}^{2} \mu_{i} F_{i} x(k)
$$

By combining (10) and (11), the augmented closed-loop system is given by:

$$
x(k+1)=\sum_{i=1}^{2} \mu_{i}\left(A_{i}+B F_{i}\right) x(k)+E w(k)
$$

In what follows, we denote $\Phi=\sum_{i=1}^{2} \mu_{i}\left(A_{i}+B F_{i}\right)$, the closed loop system takes the form:

$$
x(k+1)=\Phi x(k)+E w(k)
$$

\section{A. Invariant set under state feedback}

Assume that there exists a quadratic function $V(x)=x^{T} P x$, where $P=G^{-1}$ is a symmetric positive definite matrix, that satisfies, for all $x, w$ satisfying (13), $w^{T} w \leq 1, V(x) \geq 1$, the condition [14]:

$$
V(x(k+1)) \leq V(x(k))
$$

Let us consider the reachable set $\Lambda$ defined by:

$$
\begin{aligned}
\Lambda \triangleq \quad & \left\{x\left(k^{\prime}\right) \mid x, w\right. \text { satisfying (13), } \\
& \left.x(0)=0, w^{T} w \leq 1, k^{\prime} \geq 0\right\}
\end{aligned}
$$

The set $\varepsilon_{P}$ defined by:

$$
\varepsilon_{P}=\left\{x(k) \in \mathscr{R}^{4 n} \mid x(k)^{T} \operatorname{Px}(k) \leq 1\right\},
$$

is an invariant set for system (13) with $w \in \mathscr{R}, w^{T} w \leq 1$. This means that every trajectory that starts inside $\varepsilon_{P}$ remains inside it for $k \geq 0$.

Similarly, we will define the set $\varepsilon_{\eta P}$ where $\eta \in(0,1]$ by

$$
\varepsilon_{\eta P}=\left\{x(k) \in \mathscr{R}^{4 n} \mid x(k)^{T} \eta P x(k) \leq 1\right\}
$$

The existence of such a function $V(x)$ means that the set $\varepsilon_{P}$ is an outer approximation of the reachable set $\Lambda$. 
$\varepsilon_{P}$ is also an outer approximation of the reachable set

$$
\begin{aligned}
\Lambda^{*} \triangleq \quad & \left\{x\left(k^{\prime}\right) \mid x, w\right. \text { satisfying equation (13), } \\
& \left.x(0) \in \varepsilon_{P}, w^{T} w \leq 1, k^{\prime} \geq 0\right\}
\end{aligned}
$$

In this section, the control law and the invariant set $\varepsilon_{P}$ are synthesized. This is achieved using BMI (Bilinear Matrix Inequalities) optimization method such that the system without the disturbance is asymptotically stable and at the same time, the reachable set for an initial state values inside the invariant set is contained in this invariant set.

\section{B. State feedback synthesis}

Quadratically boundedness of the closed loop system is ensured if we want to impose the condition $w(k)^{T} w(k) \leq$ 1 and $V(x(k)) \geq 1$ implies $V(x(k+1))<V(x(k))$. A slightly more conservative condition that is more amenable to LMI formulation is to impose $w(k)^{T} w(k) \leq V(x(k))$ implies $V(x(k+1))<V(x(k))$. Re-writing the state feedback gains under the form $F_{i}=Y_{i} Q_{i}^{-1}$ where $Y_{i}$ are matrices of compatible dimensions and $Q_{i}$ are full rank, the quadratic boundedness is ensured if the LMI conditions hold for $i=1,2$

$$
\left[\begin{array}{cccc}
Q_{i}^{T}+Q_{i}-G & 0 & \alpha Q_{i}^{T} & Q_{i}^{T} A_{i}^{T}+Y_{i}^{T} B^{T} \\
0 & \beta I & 0 & E^{T} \\
\alpha Q_{i} & 0 & \alpha G & 0 \\
A_{i} Q_{i}+B Y_{i} & E & 0 & G
\end{array}\right]>0
$$

and $\alpha \geq \beta>0$. Note that the relaxation inequality $Q_{i}^{T} P Q_{i}>$ $Q_{i}^{T}+Q_{i}-G$ is used.

\section{Constraints on input and state components}

In addition, it is possible to handle constraints on the control signal and the state:

$$
-\bar{u} \leq u(k) \leq \bar{u}, \quad-\bar{\Psi} \leq \Psi x_{e}(k+1) \leq \bar{\Psi}, \quad \forall t \geq 0
$$

where $\bar{u}:=\left[\bar{u}_{1}, \bar{u}_{2}, \ldots, \bar{u}_{n}\right]^{T}>0$, with $\bar{u}_{j}>0, j=1, \ldots, n$, and $\bar{\Psi}:=\left[\bar{\Psi}_{1}, \bar{\Psi}_{2}, \ldots, \bar{\Psi}_{q}\right]^{T}$ with $\bar{\Psi}_{m}>0, m=1, \ldots, q, \Psi \in$ $\mathscr{R}^{q \times(4 n)}$ and $q$ is the number of imposed constraints. Note that the bounds are provided separately on each state variables or a combination of them [18].

Let us define $\xi_{j}$ as the $j$-th row of the $n$ ordered identity matrix. For a pre-specified scalar $\eta \in(0,1]$, the quadratic boundedness property ensures that if $x(0) \in \varepsilon_{\eta P}$, then $x(k) \in$ $\varepsilon_{\eta P}, \forall k \geq 0$, thus $\forall w(k)$ such that $w(k)^{T} w(k) \leq 1$

$$
\begin{aligned}
& \max _{k \geq 0}\left|\xi_{j} u(k)\right|^{2}=\max _{k \geq 0}\left|\xi_{j} \sum_{i=1}^{2} \mu_{i} F_{i} x(k)\right|^{2} \\
& \leq \max _{k \geq 0}\left\|\left[\sum_{j=1}^{2} \mu_{i} F_{i}\right][\eta P]^{-1 / 2}\right\|^{2} \times\left\|[\eta P]^{1 / 2}[x(k)]\right\|^{2} \\
& \leq\left\|\left[\sum_{i=1}^{2} \mu_{i} F_{i}\right][\eta P]^{-1 / 2}\right\|^{2}
\end{aligned}
$$

If a symmetric matrix $Z$ exists such that

$$
Z-\left[\sum_{i=1}^{2} \mu_{i} F_{i}\right]^{T}[\eta P]^{-1}\left[\sum_{i=1}^{2} \mu_{i} F_{i}\right] \geq 0
$$

and $Z_{j j}<\bar{u}_{j}^{2}, j=1, \ldots, n$, then $|u(k)|<\bar{u}$. By applying the Schur complement, one can first achieve

$$
\left[\begin{array}{cc}
\eta P & \left(\sum_{i=1}^{2} \mu_{i} F_{i}\right)^{T} \\
\sum_{i=1}^{2} \mu_{i} F_{i} & Z
\end{array}\right] \geq 0
$$

which could be transformed into

$$
\left[\begin{array}{cc}
Q_{i}^{T}+Q_{i}-\eta G & Y_{i}^{T} \\
Y_{i} & Z
\end{array}\right] \geq 0
$$

A similar procedure can be applied for the constraints on the state variables. Define $\xi_{j}$ as the $\mathrm{j}$-th row of the q-ordered identity matrix. Then

$$
\begin{aligned}
& \max _{k \geq 0}\left|\xi_{j} \Psi x(k+1)\right|^{2}= \\
& \max _{k \geq 0}\left|\xi_{j} \Psi\left[\begin{array}{ll}
\Phi & E
\end{array}\right]\left[\begin{array}{c}
x(k) \\
w(k)
\end{array}\right]\right|^{2} \\
& \leq 2\left\|\xi_{j} \Psi\left[\begin{array}{ll}
\Phi & E
\end{array}\right]\left[\begin{array}{cc}
\eta P & 0 \\
0 & I
\end{array}\right]^{-1 / 2}\right\|^{2}
\end{aligned}
$$

If a symmetric matrix $\Xi$ exists such that

$$
\Xi-2 \Psi\left[\begin{array}{ll}
\Phi & E
\end{array}\right]\left[\begin{array}{cc}
\eta P & 0 \\
0 & I
\end{array}\right]^{-1}\left[\begin{array}{l}
\Phi^{T} \\
E^{T}
\end{array}\right] \Psi^{T} \geq 0
$$

$\Xi_{m m} \leq \bar{\Psi}_{m}^{2}, m=1, \ldots, q$, then $\|\Psi x(k+1)\|<\bar{\Psi}, \forall k \geq 0$. By applying the Schur complement, it can be shown that (24) is equivalent to

$$
\left\{\begin{array}{c}
{\left[\begin{array}{ccc}
Q_{i}^{T}+Q_{i}-\eta G & * & * \\
0 & I & * \\
\sqrt{2} \Psi\left(A_{i} Q_{i}+B Y_{j}\right) & \sqrt{2} \Psi E & \Xi
\end{array}\right] \geq 0} \\
\Xi_{m m} \leq \bar{\Psi}_{m}^{2}, m=1, \ldots, q,
\end{array}\right.
$$

\section{Avoiding entering the collision zone}

The condition that the vehicles do not enter the collision zone (see Fig. 1) can be written as $\left|\delta_{i}\right| \leq \delta^{M}$. Moreover, if the relative speed is limited, $\left|v_{i}^{r}\right| \leq v_{r}^{M}$, and the required longitudinal acceleration/deceleration is remaining within acceptable comfort values $\left|a_{i}\right| \leq a^{M}$, the three above constraints define a parallelepiped denoted by $L\left(F^{M}\right)$. A "safe following" is then defined by $x_{e} \in L\left(F^{M}\right)$. The matrix $F^{M}$ is given by:

$$
F^{M}=\left(\begin{array}{c}
f_{1}^{M} \\
f_{2}^{M} \\
\vdots \\
f_{n}^{M}
\end{array}\right)
$$

where $f_{i}^{M}=\left[0_{1 \times(4(i-1)}, \delta^{M}, v_{r}^{M}, a^{M}, 0,0_{1 \times(4(n-i)}\right]$

$$
L\left(F^{M}\right) \triangleq\left\{x_{e} \in \mathscr{R}^{4 n}:\left|f_{i}^{M} x_{e}\right| \leq 1, i=1,2, n\right\} .
$$

The conditions ensuring that the invariant set $\varepsilon_{P}$ is in inside this hypercube are [19]:

$$
f_{i}^{M} G\left(f_{i}^{M}\right)^{T}-1<0, i=1,2,3 .
$$

\section{E. Optimization problem for control synthesis}

We are now able to provide the BMI optimization problem with decision variables $G, Y_{j}, \Theta_{i j},(i, j=1,2), \Xi$ and $\alpha$, which ends with the control gain vectors $F_{1}$ and $F_{2}$ :

$$
\begin{array}{ccc}
\min & \operatorname{trace}(G) & \\
G \succ 0, & \alpha>0, \\
\text { eq. }(19), & \text { eq. }(22), \\
& \text { LMIs from eq.(25), } & \text { eq. }(28)
\end{array}
$$


This BMI optimization problem is solved using the software developed by the authors of [20]. The trace of matrix $G$ is minimized in order to reduce the excursions from the equilibrium point. Note that it is also possible to weight the magnitude of the maximum admissible disturbance which is also a design parameter.

\section{Simulation Results}

The control law is now tested in a simulation environment which includes different scenarios.

\section{A. Numerical values}

The numerical values defining the "safe following" zone are chosen using the same idea but with different proportions: $v_{r}^{M}=0.25 v_{f}, \delta^{M}=0.3 s_{d}$. The optimization problem is solved while the BMI and LMI constraints are found feasible.

The assistance is mainly designed in order to operate in soft braking and acceleration modes. The brake and the acceleration bounds are chosen equal in absolute values to $u_{\max }=6 \mathrm{~m} / \mathrm{s}^{2}$, while the maximum brake magnitude of the leading vehicle is $\gamma_{l}^{\max }=8 \mathrm{~m} / \mathrm{s}^{2}$. The solutions for the BMI optimization problems from eq. (29) are obtained using the Yalmip software on MATLAB.

\section{B. Simulation results for braking and acceleration profiles}

In the following, for sake of clarity, the vehicles length and the minimum spacing at stopping $s_{0}$ are chosen equal to zero. The distance at standing is set to $s_{0}=5 \mathrm{~m}$ and the time headway to $h=1.2 \mathrm{sec}$. Vehicles index are numbered from 0 to 3 . The initial vehicle positions are $x_{0}(0)=60 \mathrm{~m}, x_{1}(0)=50 \mathrm{~m}$, $x_{2}(0)=20 m, x_{3}(0)=0 m$. The initial cruising speed is set to $v_{i}(0)=15 \mathrm{~m} / \mathrm{s}$. So the initial headway distances are thus of $18 m$. There is an initial offset of $2 m$ between two vehicles. The leading vehicle acceleration profile is depicted on Fig. 2. It alternates braking, cruising and acceleration profiles. Therefore, these profiles include stop-and-go scenario. In practice, the estimation of the leader vehicle acceleration could be affected by noise or a bias. Thus, the previous maneuver is simulated with non perfect measurement of the leader vehicle assuming a zero mean Gaussian additive noise signal with variance equal to 0.1 and a bias equal to $0.1 \mathrm{~m} / \mathrm{s}^{2}$. Fig. 3 shows four plots corresponding to the 3 vehicles speeds. one can notice that all the three other speeds converge toward the leading vehicle speed with a maximum delay of three seconds after it has reached its cruising speed. This convergence is also preserved while performing stop and go maneuver between time $120 \mathrm{sec}$ and $140 \mathrm{sec}$. The following vehicles speeds do not exceed leading vehicle speed. Corresponding acceleration profiles are shown in Fig 3 (c). Maximum values are always under $5 \mathrm{~m} / \mathrm{s}^{2}$ both during braking and acceleration. Headway distances are given in Fig. 3(a). Multiplying cruising speed values of Fig. 3(b) with the selected headway time of $1.2 \mathrm{sec}$ allows to verify if the headway distances converge toward the desired values. All the responses are smooth, the control law achieves a high quality filtering of the noise. For example, headway distance is of about $30 \mathrm{~m}$ at $t=60 \mathrm{sec}$, while the cruising

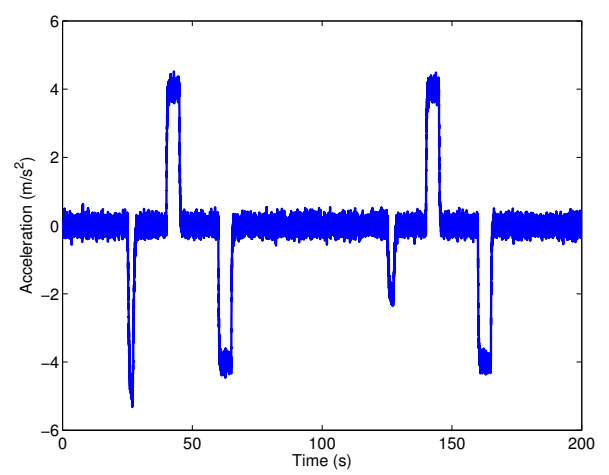

Fig. 2. Leading vehicle acceleration profile.

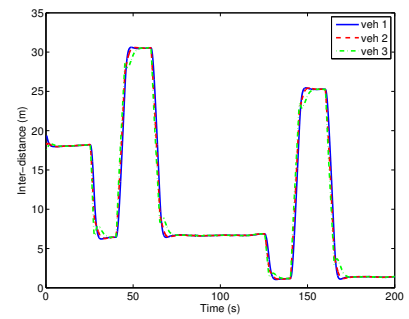

(a) Inter-distance

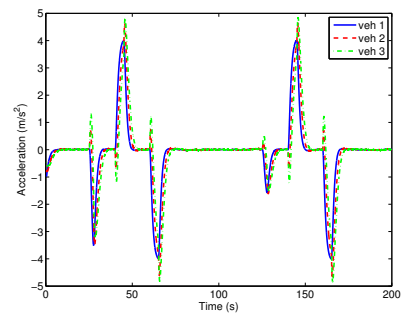

(c) Acceleration

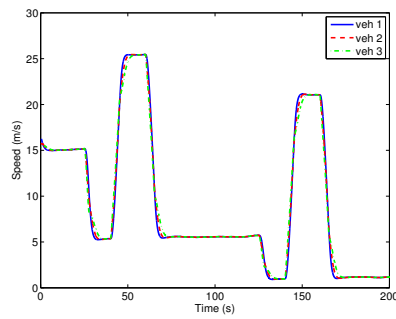

(b) Speed

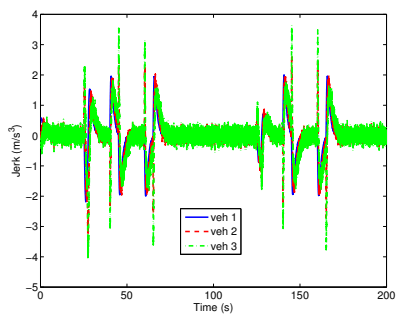

(d) Jerk
Fig. 3. Inter-distance, velocities, acceleration and jerk for a given leader acceleration profile.

speed if $25.4 \mathrm{~m} / \mathrm{s}$. Finally, Fig. 3(d) provides the jerk of the three vehicles of the string. The jerk of the first vehicle is bounded by $\left(<4 \mathrm{~m} / \mathrm{s}^{3}\right)$. The noise during the acceleration of the leading vehicle appears on the following vehicles, it is however smoothed when we go upstream.

Finally, Fig. 4 provides the plot of the two relative speeds between the vehicles of the string. It is maintained below $2 \mathrm{~m} / \mathrm{s}$ for its absolute value, which is under the maximum allowed value $v_{r}^{M}=3.75 \mathrm{~m} / \mathrm{s}$.

\section{Testing for different headway time values}

Now suppose that we starting with a time headway of $1.2 \mathrm{sec}$, the infrastructure manager decides to linearly increase it until $2.5 \mathrm{sec}$ between $[20,30] \mathrm{sec}$ and holds it for a time duration of $70 \mathrm{sec}$. Thus it is linearly decreased again towards $1.5 \mathrm{sec}$ in the interval $[160,180] \mathrm{sec}$ and then maintained to this value (Fig. 5). The leading vehicle acceleration profile is maintained identical. Figure 6 shows the corresponding experienced inter-distance. We can notice that the control law introduces adaptation for this variable 


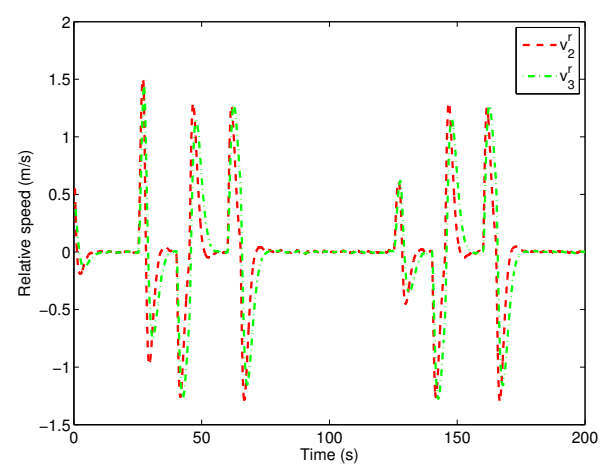

Fig. 4. Vehicles relative speeds inside the string.

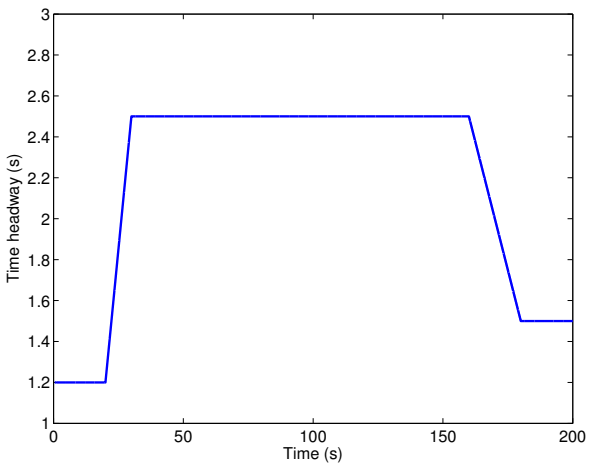

Fig. 5. Variable time headway profile.

headway profile for the all the vehicles in the string. In addition, the adaptation is smooth.

\section{CONCLUSION}

This paper presents the design and the simulation test of a vehicle following control law for a string of four highly cooperative vehicles with variable headway time handling. A control law based on linking Lyapunov functions and invariant sets for multi-model systems with LMI and BMI optimization is suggested. This approach ensures four important features: asymptotic convergence of the vehicles trajectories to the reference headway distance with variable headway time, guarantees that the vehicles remain inside a "safe following" zone, and a bounded control input for all bounded leading vehicle acceleration. Simulation tests

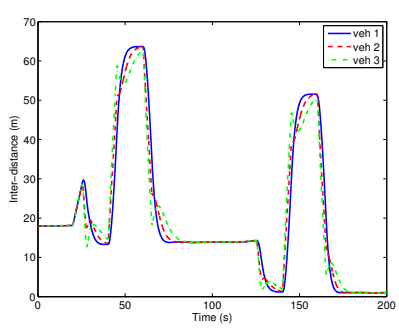

(a) Inter-distance

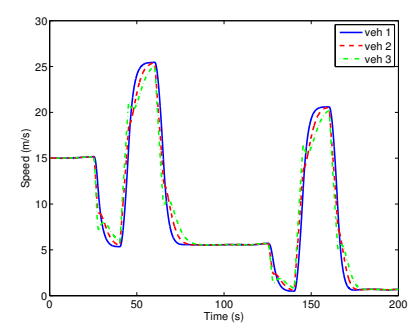

(b) Speed
Fig. 6. Inter-distance, velocities for a variable headway time with the same leader acceleration profile. show that vehicles behaviour agrees with the specifications. The controller tunes automatically when varying the time headway. It has been verified that safety and comfort for the entire range of speed are achievable. The approach offers also the flexibility to be applicable even in autonomous or highly cooperative mode. In fact, each vehicle could switch at any moment to a new controller for which only onboard sensors (typically ACC sensors) are used. The field test of the proposed strategy on prototype vehicles is the object of a future work.

\section{REFERENCES}

[1] X. Lingyun, G. Feng, "A comprehensive review of the development of adaptive cruise control systems", Vehicle System Dynamics, vol. 48, no.10, pp. 1167-1192, 2010.

[2] V. Milanes, J. Villagra, J. Godoy, C. Gonzalez, "Comparing Fuzzy and Intelligent PI Controllers in Stop-and-Go Manoeuvres", IEEE Trans. on Control Systems Technology, vol. 99, no. 1, pp. 1-9, 2011.

[3] P. Varaiya, "Smart cars on smart roads: problems of control", IEEE Trans. Automatic Control, 38:195-207, 1993.

[4] D. Yanakiev and I. Kanellakopoulos, "Longitudinal control of heavyduty vehicles for automated highway systems", Proc. 1995 American Ccontrol Conference, Seattle, WA, pages 3096-3100, 1995.

[5] J. K. Hedrick, D. H. McMahon, V. K. Narendran, and D. Swaroop, "Longitudinal vehicle controller design for ivhs systems", Proc. 1991 American Ccontrol Conference, Boston, MA, pages 3107-3112, 1991.

[6] E. Chan, E. Coelingh, T. Robinson, "Operating Platoons On Public Motorways: An Introduction To The SARTRE Platooning Programme", 17th World Congress on Intelligent Transport Systems, Busan, Korea, 2010.

[7] J. Jairo-Martinez and C. Canudas-de-Wit, "A Safe Longitudinal Control for Adaptive Cruise Control and Stop-and-Go Scenarios", IEEE Trans. on Cont. Sys. Technology, vol. 15, no. 2, pp. 246-258, 2007.

[8] G. Rödönyi, P. Gáspár, J. Bokor, Sz. Aradi, Z. Hankovszki, R. Kovács and L. Palkovics, "Analysis and experimental verification of faulty network modes in an autonomous vehicle string", 20th Mediterranean Conference on Control and Automation, Barcelona, Spain, 2012.

[9] G. Rödönyi, P. Gáspár, J. Bokor, L. Palkovics, "Guaranteed peaks of spacing errors in an experimental vehicle string", 7th IFAC Symposium on Robust Control Design, 2012.

[10] P. Fernandes, U. Nunes, "Algorithms for Management of a MultiPlatooning System of IVC-enabled Autonomous Vehicles, with High Traffic Capacity", 14th International IEEE Conference on Intelligent Transportation Systems, Washington, 2011.

[11] G.J.L. Naus, J.Ploeg, M.J.G.VandeMolengraft, W.P.M.H. Heemels, M.Steinbuch, "Design and implementation of parameterized adaptive cruise control: An explicit model predictive control approach", Control Engineering Practice, vol. 18, no. 8, pp. 882-892, 2010.

[12] S. Moon, I. Moon, K. Yi, "Design, tuning and evaluation of a fullrange adaptive cruise control system with collision avoidance", Control Engineering Practice, vol. 17, no. 4, pp. 442-455, 2009.

[13] W. Prestl, T. Sauer, J. Steinle, O. Tschernoster, "The BMW active cruise control ACC", SAE tech. paper (2000-01-0344), 2000.

[14] F. Blanchini, S. Miani, "Set-theoretic methods in control", Sys. \& Cont.: Found. \& App., Birkhauser, Boston-Basel-Berlin, 2008.

[15] Z.P. Jiang, Y and Wang, "Input-to-state stability for discrete-time nonlinear systems", Automatica, Vol. 37, no. 6, pp.857-869, 2001.

[16] N. Minoiu, S. Mammar, N. Netto and B. Lusetti, "Driver steering assistance for lane departure avoidance based on hybrid automata and on composite Lyapunov function", IEEE Trans. on Intelligent Transportation Systems, vol. 11, no. 1, pp. 28-39, 2010.

[17] L. Nouvelière and S. Mammar, "Experimental vehicle longitudinal control using a second order sliding modes technique", Control Engineering Practice, vol. 15, no. 8, pp. 943-954, 2007.

[18] B. Ding, "Quadratic boundedness via dynamic output feedback for constrained nonlinear systems in Takagi-Sugeno's form", Automatica, vol. 45, no. 9, pp. 2093-2098, 2009.

[19] S. Boyd, L. El Ghaoui, E. Feron and V. Balakrishnan, "Linear Matrix Inequalities in System and Control Theory", SIAM, Phil., 1994.

[20] M. Kocvara and M. Stingl, "PENBMI Users Guide (Version 2.1), software manual", PENOPT GbR, Hauptstrasse 31A, 91338 Igensdorf OT Stckach, Germany, 2006. 